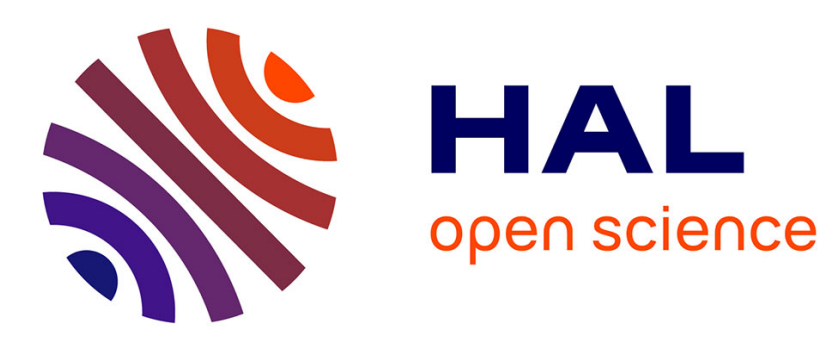

\title{
Dual-Band Vector Sensor for Direction of Arrival Estimation of Incoming Electromagnetic Waves
}

Jimmy Lominé, Christophe Morlaas, Christophe Imbert, Hervé Aubert

\section{To cite this version:}

Jimmy Lominé, Christophe Morlaas, Christophe Imbert, Hervé Aubert. Dual-Band Vector Sensor for Direction of Arrival Estimation of Incoming Electromagnetic Waves. IEEE Transactions on Antennas and Propagation, 2015, 63 (8), pp.3662 - 3671. 10.1109/TAP.2015.2435039 . hal-01196813

\section{HAL Id: hal-01196813 \\ https://hal-enac.archives-ouvertes.fr/hal-01196813}

Submitted on 21 Sep 2015

HAL is a multi-disciplinary open access archive for the deposit and dissemination of scientific research documents, whether they are published or not. The documents may come from teaching and research institutions in France or abroad, or from public or private research centers.
L'archive ouverte pluridisciplinaire HAL, est destinée au dépôt et à la diffusion de documents scientifiques de niveau recherche, publiés ou non, émanant des établissements d'enseignement et de recherche français ou étrangers, des laboratoires publics ou privés. 


\title{
Dual-Band Vector Sensor for Direction of Arrival Estimation of Incoming Electromagnetic Waves
}

\author{
Jimmy Lominé, Christophe Morlaas, Christophe Imbert, and Hervé Aubert, Senior Member, IEEE
}

\begin{abstract}
In this paper, a dual-band vector sensor using antennas spatially distributed and diversely polarized for estimating the direction of arrival (DoA) of electromagnetic fields in two frequency bands is reported for the first time. To cover the two bands the sensor is composed of six small dual-band radiating elements: three half-loops and three monopoles. The accuracy of the DoA estimation is computed from the well-known multiple signal classification algorithm. A dual-band vector sensor prototype is designed and manufactured for operating in two global system for mobile communications frequency bands. Measurement results are reported for experimental validation purpose. The extension of the proposed methodology to the design of multiband vector sensors is finally discussed.
\end{abstract}

Index Terms-Antenna diversity, direction of arrival (DoA) estimation, multifrequency antennas, ultrahigh frequency (UHF) antennas.

\section{INTRODUCTION}

$\mathbf{T}$ HE PURPOSE of radio direction finders (RDF) is to estimate the direction of arrival (DoA) of incoming waves radiated by electromagnetic (EM) sources. An RDF can be used for defense as well as civil applications such as radio astronomy, navigation systems, and rescue devices [1]. In order to estimate the DoA of incoming EM-fields, the spatial phase diversity resulting from the spatial distribution of the sensor antennas [2] or the polarization diversity of the sensor [3] are usually used. The combination of these two well-known approaches has also been proposed to improve the accuracy of the DoA estimation [4]-[10]. The DoA estimation based on the spatial diversity consists of measuring the incoming EM-fields by using a single-polarized array of distributed elements whereas the use of the diversity of polarization is based on the measurement of the EM-field components by using, e.g., a vector sensor composed of six antennas: three orthogonal electric dipoles and three orthogonal magnetic dipoles [11]. Nevertheless, following [10], [12]-[18], the measurement of only three EM-field components seems to be sufficient for the accurate DoA estimation of transverse EM-fields in the

Manuscript received November 19, 2014; revised March 25, 2015; accepted April 24, 2015. Date of publication May 19, 2015; date of current version July 31, 2015. This work was supported by Rockwell Collins France under contract ENAC/2012/DR/URI/IR/00020

J. Lominé and C. Imbert are with the Department of Navigation and Surveillance, Rockwell Collins France, 31701 Blagnac, France (e-mail: jimmy.lomine@ rockwellcollins.com).

C. Morlaas is with ENAC, TELECOM-EMA, Toulouse F-31055, France, and also with the University of Toulouse, Toulouse F-31400, France.

H. Aubert is with LAAS-CNRS, MINC, Toulouse F-31400, France, and also with the University of Toulouse, Toulouse F-31400, France.

Color versions of one or more of the figures in this paper are available online at http://ieeexplore.ieee.org.

Digital Object Identifier 10.1109/TAP.2015.2435039 three-dimensional (3-D) half-space. In [10], single-band vector sensors using only three co-located or distributed antennas are reported for estimating the DoA of incoming transverse magnetic (TM) EM-fields in the 3-D half-space.

In order to estimate the DoA at several frequencies without increasing the number of vector sensors, RDF may incorporate wideband or multiband radiating elements. For the first time, at least to the authors' knowledge, a vector sensor is reported for estimating the DoA of EM-fields in the 3-D half-space and in two frequency bands using antennas spatially distributed and diversely polarized.

In this paper, a ultrahigh frequency (UHF) dual-band vector sensor allowing the DoA estimation of incoming TM EM-fields in the 3-D half-space and in two frequency bands is designed and its performances are measured. This first sensor is composed of three dual-band loaded half-loops and three dual-band folded-monopoles placed on a metallic plate. For experimental validation purpose, a prototype of this sensor has been designed for operating in the two following global system for mobile (GSM) communications frequency bands: $(890-960 \mathrm{MHz})$ and $(1710-1880 \mathrm{MHz})$. The accuracy of the DoA estimation is computed here from the multiple signal classification (MUSIC) algorithm [19].

This paper is organized as follows. Section II defines criteria for evaluating the DoA estimation performances of vector sensors. In Section III, the single-band vector sensor recently reported by the authors in [10] is briefly described and its accuracy for estimating the DoA is reported. Throughout this paper this single-band sensor is considered as the reference sensor. In section IV, an original topology of a dual-band vector sensor is proposed. The performances of this novel sensor for DoA estimation are predicted in Section V from full-wave EM simulations. The experimental validation is reported in Section VI and shows that the accuracy of the DoA estimation is better in the two frequency bands than one provided by the reference sensor. Finally, Section VII discusses the extension of the proposed methodology to the design of multiband vector sensors.

\section{Typical TeChnical Requirement for Vector Sensors Applied to the DoA Estimation}

In an homogeneous, linear, isotropic and lossless medium the propagation direction of an EM-wave is given by the direction of the wave vector $\boldsymbol{k}(\phi, \theta)$. The DoA of an incoming EM-field in the 3-D half-space is then defined by the azimuth angle $\phi \in$ $\left[0^{\circ} ; 360^{\circ}\right]$ and the elevation angle $\theta \in\left[0^{\circ} ; 90^{\circ}\right]$ of $\boldsymbol{k}(\phi, \theta)$ (see Fig. 1). 


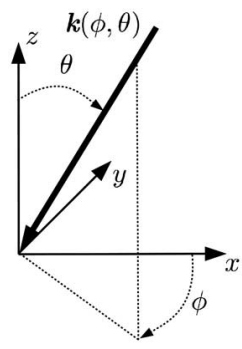

Fig. 1. Azimuth $(\phi)$ and elevation $(\theta)$ angles defining the direction of the incoming EM-wave in the cartesian coordinate system Oxyz.

Basically a vector sensor allows measuring simultaneously the EM-field components for deriving the estimation of the DoA [11]. Moreover the sensor elements can be spatially distributed for improving the accuracy of the DoA estimation [20]. Let $\boldsymbol{X}(\phi, \theta, \gamma, \eta) \in \mathbb{C}^{M}$ be the vector of the EM-field components measured by a sensor incorporating $M$ antennas. This vector may be written as follows [11]:

$$
\boldsymbol{X}=\boldsymbol{D}(\phi, \theta, \gamma, \eta) \cdot \boldsymbol{S}+\boldsymbol{W}
$$

where $\boldsymbol{D}(\phi, \theta, \gamma, \eta) \in \mathbb{C}^{M, K}$ is the matrix of the steering vectors. These vectors are designated by $\left\{\boldsymbol{d}_{k}(\phi, \theta, \gamma, \eta)\right\}_{k=1, \ldots, K}$ and are associated with the $\mathrm{K}$ incoming EM-waves. The polarization parameters, denoted by $\gamma \in\left[0^{\circ} ; 90^{\circ}\right]$ and $\eta \in$ $\left[-180^{\circ} ; 180^{\circ}\right]$, refer to the auxiliary polarization angle and the polarization phase difference, respectively. $\boldsymbol{S} \in \mathbb{C}^{K}$ is the vector of the complex envelopes of the $\mathrm{K}$ incoming signals written $S_{k}=\left|s_{k}\right| e^{j \psi_{k}}$, where $s_{k}$ and $\psi_{k}$ are the amplitude and phase of the kth signal. $\boldsymbol{W} \in \mathbb{C}^{M}$ is an uncorrelated Gaussian noise vector with a zero-mean. In this paper, $\mathrm{K}=1, \gamma=90^{\circ}$, and $\eta=0^{\circ}$.

Based on (1) the MUSIC algorithm is applied for computing the DoA [19] as follows:

1) Step 1: The noise subspace is determined from the eigenvalue decomposition of the covariance matrix of $\boldsymbol{X}(\phi, \theta)$;

2) Step 2: In each direction of the 3-D half-space, the steering vector $\boldsymbol{d}(\phi, \theta)$ is projected onto the estimated noise subspace;

3) Step 3: The DoA is derived from the computation of the lowest projection value.

As shown in [21], a calibration process is needed to take into account the amplitude and phase distortions between the sensor outputs due to eventual radiation perturbations. Several calibration methods have been proposed [22]-[24]. In this work, the calibration is achieved by using the (simulated or measured) radiating patterns of each sensor antenna. The electric field radiated by each antenna with the same input power is computed. Then the steering vector is computed from these electric fields with $\boldsymbol{d}_{k}(\phi, \theta)=E_{k}(\phi, \theta)$ where $E_{k}(\phi, \theta)$ denotes the electric field radiated by the $k$ th antenna.

For designing a vector sensor, the technical requirement on the following key sensor descriptors must be specified:

1) The angular ambiguity. An angular ambiguity occurs when MUSIC algorithm is not able to discriminate DoAs that are associated with collinear steering vectors. Thus, the risk of the angular ambiguity is analyzed as follows [25]:

$$
\alpha\left(\phi_{1}, \theta_{1}, \phi_{2}, \theta_{2}\right)=\cos ^{-1} \frac{\left|\boldsymbol{d}\left(\phi_{1}, \theta_{1}\right)^{*} \cdot \boldsymbol{d}\left(\phi_{2}, \theta_{2}\right)\right|}{\left\|\boldsymbol{d}\left(\phi_{1}, \theta_{1}\right)\right\| \cdot\left\|\boldsymbol{d}\left(\phi_{2}, \theta_{2}\right)\right\|}
$$

where $\alpha \in\left[0^{\circ} ; 90^{\circ}\right]$ denotes the angle (modulo $90^{\circ}$ ) between the two steering vectors $\boldsymbol{d}\left(\phi_{1}, \theta_{1}\right)$ and $\boldsymbol{d}\left(\phi_{2}, \theta_{2}\right)$. An ambiguity occurs when $\alpha=0^{\circ}$. The ambiguity analysis allows deriving the maximum separation distance between the sensor antennas, denoted here by $d_{\mathrm{AMB}}$, for avoiding angular ambiguities [10]. For each pair $\left(\phi_{i}, \theta_{i}\right)$ and $\left(\phi_{j}, \theta_{j}\right)$ with $i \neq j$ the angle $\alpha$ is computed, and the minimum value of $\alpha$ is plotted in a 3-D polar plot to obtain the ambiguity spectrum.

2) The accuracy of the DoA estimation. The accuracy is derived from the angular distance between the estimated $\operatorname{DoA}(\hat{\phi}, \hat{\theta})$ and the real DoA $(\phi, \theta)$. This angular distance is defined by Maral [26]

$$
\begin{aligned}
\Delta a(\phi, \theta)= & \cos ^{-1}(\cos (\theta) \cos (\hat{\theta}) \\
& +\sin (\theta) \sin (\hat{\theta}) \cos (\phi-\hat{\phi})) .
\end{aligned}
$$

The root-mean-square (rms) error, denoted by $\Delta a_{\mathrm{rms}}(\phi, \theta)$, is then computed by considering $L$ estimations per DoA. The resulting rms is then given by

$$
\Delta a_{\mathrm{rms}}(\phi, \theta)=\sqrt{\frac{1}{L} \sum_{i=1}^{L}|\Delta a(\phi, \theta)|^{2}} .
$$

3) The sensitivity of the vector sensor. The sensitivity is the minimum incident power density $P_{r}$ relative to a noise power level $P_{n}$ for obtaining a specified rms error $\Delta a_{\mathrm{rms}}(\phi, \theta)$. In this paper, the sensitivity is related to the incoming signal power density to noise ratio PNR given by

$$
\mathrm{PNR}=\frac{P_{r}}{P_{n}} .
$$

A typical noise figure of $\mathrm{NF}=10 \mathrm{~dB}$ in a bandwidth $\mathrm{BW}=200 \mathrm{kHz}$ (channel bandwidth in the GSM standard) is used here that yields $P_{n}=-111 \mathrm{dBm}$. The sensitivity $P_{\text {sensi }}$ is obtained for $\Delta a_{\mathrm{rms}}(\phi, \theta)=5^{\circ}$ in the 3-D half-space. $P_{n}$ is the same for all DoAs (the noise in the propagation channel noise is assumed to be lower than the noise provided by the receiver and consequently, PNR is here spatially invariant).

4) The size of the vector sensor. The sensor has to use a number of antennas as low as possible for reducing the number of receiving channels and minimizing the size of the vector sensor. Separation distance between the antennas may be useful for achieving high accuracy in the DoA estimation [20] and high sensor sensitivity. However large distances may create undesirable angular ambiguities. 


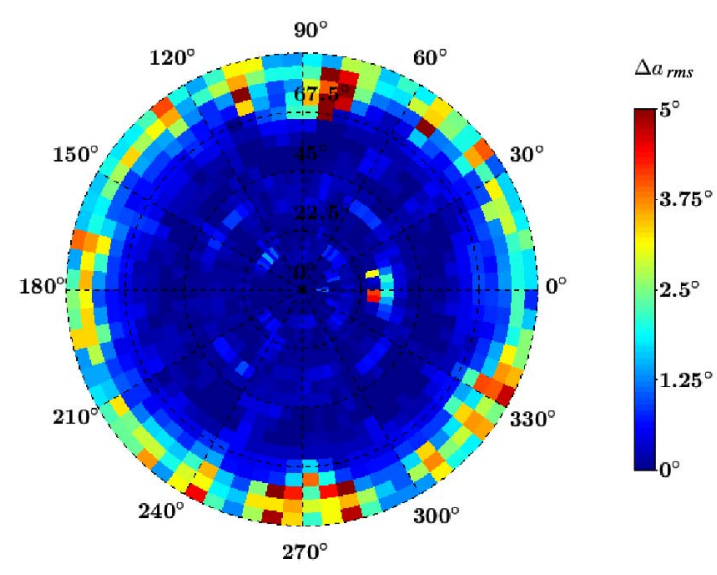

Fig. 2. Simulated $\Delta_{a \mathrm{rms}}(\phi, \theta)$ at $2400 \mathrm{MHz}$ as a function of $\phi$ and $\theta$ obtained with the initial vector sensor located on a $800 \times 800 \mathrm{~mm}$ metallic support with PNR $=46 \mathrm{~dB} \mathrm{~m}^{-2}, N=100$, and $L=20$. The elevation angles $\theta$ are given in the radial axis.

\section{Reference Single-BAnd Vector Sensor}

A single-band vector sensor has been proposed in [10]. It is composed of three equidistant radiating elements, located in the $O x y$ plane: one small wire antenna for measuring the $z$-component of the electric field (E-field) and two small halfloops for measuring the $x$ - and $y$-components of the magnetic field (H-field). It has been shown that the measurement of these three components is sufficient for estimating the DoA of TM incoming EM-fields in the 3-D half-space. The accurate estimation of the DoA from the MUSIC algorithm may be obtained by properly choosing the distance of the antennas from the center of the sensor. An ambiguity analysis performed in [10] defined the maximum distance $d_{\mathrm{AMB}}=0.17 \lambda$.

The vector sensor introduced in [10] has been simulated through a full-wave EM software (HFSS). A $800 \times 800 \mathrm{~mm}$ metallic plate has been used for modeling the impact of a large metallic support on the sensor performances. The estimation conditions are: an operating frequency $f=2400 \mathrm{MHz}$, an incident power density $P_{r}=-95 \mathrm{dBW} \mathrm{m}^{-2}$ which corresponds to $\mathrm{PNR}=46 \mathrm{~dB} \mathrm{~m}^{-2}, \mathrm{~N}=100$ snapshots per DoA estimation, and $\mathrm{L}=20$ estimations per DoA. The simulation was performed for $\phi \in\left[0^{\circ} ; 360^{\circ}\right]$ by step of $5^{\circ}$ and for $\theta \in\left[0^{\circ} ; 90^{\circ}\right]$ by step of $5^{\circ}$. The simulated $\Delta_{a \mathrm{rms}}(\phi, \theta)$ is given in Fig. 2 as a function of azimuth $\phi$ and elevation $\theta$ angles.

The estimation is accurate in all the 3-D half-space except for DoAs close to $\theta=90^{\circ} \forall \phi$, where the maximum rms error is $5.5^{\circ}$. This error is due to the lack of dynamic on the E-field component measured by the monopole in these directions (the sensitivity is $P_{\text {sensi }}=-95 \mathrm{~dB} \mathrm{~m}^{-2}$ ). Throughout this paper, this single-band sensor is our reference vector sensor.

\section{DUAL-BANd Vector SENSOR}

\section{A. Topology of the Dual-Band Vector Sensor}

An original topology of vector sensor is proposed in Fig. 3 to improve the DoA estimation performances of the reference sensor in two frequency bands $A$ and $B$. The lowest frequency in $A$ is higher than the highest frequency in $B$. A broadband sensor

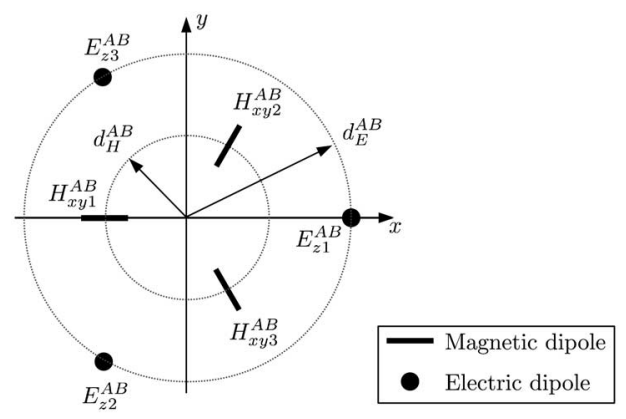

Fig. 3. Proposed topology of the dual-band vector sensor.

could be used instead of a multiband sensor to cover these two frequency bands. However, the advantage of a multiband sensor is that the gain of the sensor antennas is easier to control and to enhance at given operating frequencies.

The number of antennas is here $M=6$. Three dual-band magnetic dipoles $H_{x y i}^{A B}$ are used for measuring the $\mathrm{H}$-field along the $x$ - and $y$-axes and three dual-band electric dipoles $E_{z i}^{A B}$ are chosen for measuring the E-field along the $z$-axis ( $i$ is the antenna index). The dipoles are equidistant and located on two circles of radius $d_{H}^{A B}$ and $d_{E}^{A B}$. The proposed sensor topology provides the following advantages:

1) The EM coupling between adjacent magnetic and electric dipoles can be minimized by adjusting $d_{E}^{A B}=2 d_{H}^{A B}$. Indeed, the electric dipoles are located in the zero of the magnetic dipoles radiation.

2) The E-field component measured along the z-axis is performed at three different positions for improving the DoA estimation performances at elevation angles close to $\theta=90^{\circ}$.

3) The use of dual-band elements instead of single-band elements allows reducing the number of antennas and avoiding undesirable modification of the radiation patterns of high frequency antennas due to the masking effects caused by the large-sized antennas operating at the lower frequencies.

4) The rotation symmetry allows to obtain the same DoA estimation performances every $120^{\circ}$ in azimuth.

\section{B. Ambiguity Analysis}

An ambiguity analysis is carried out to define the largest acceptable separation distance $d_{\mathrm{AMB}}$. This analysis is performed here by neglecting the eventual EM coupling between the constitutive antennas. The ambiguity spectrum is computed as a function of the separation distance between dipoles. In TM polarization, the steering vector $\boldsymbol{d}(\phi, \theta)$ can be written as

$$
\boldsymbol{d}(\phi, \theta)=\left[\begin{array}{c}
-\sin (\theta) \\
-\sin (\theta) \\
-\sin (\theta) \\
-\cos (\phi) \\
\frac{\sqrt{3}}{2} \sin (\phi)-\frac{1}{2} \cos (\phi) \\
\frac{\sqrt{3}}{2} \sin (\phi)+\frac{1}{2} \cos (\phi)
\end{array}\right] \odot\left[\begin{array}{c}
e^{-j \boldsymbol{k} \cdot \boldsymbol{r}_{E_{z 1}^{A B}}} \\
e^{-j \boldsymbol{k} \cdot \boldsymbol{r}_{E_{z 2}^{A B}}} \\
e^{-j \boldsymbol{k} \cdot \boldsymbol{r}_{E_{z 3}^{A B}}} \\
e^{-j \boldsymbol{k} \cdot \boldsymbol{r}_{H_{x y 1}^{A B}}} \\
e^{-j \boldsymbol{k} \cdot \boldsymbol{r}_{H_{x y 2}^{A B}}} \\
e^{-j \boldsymbol{k} \cdot \boldsymbol{r}_{H_{x y 3}^{A B}}}
\end{array}\right]
$$




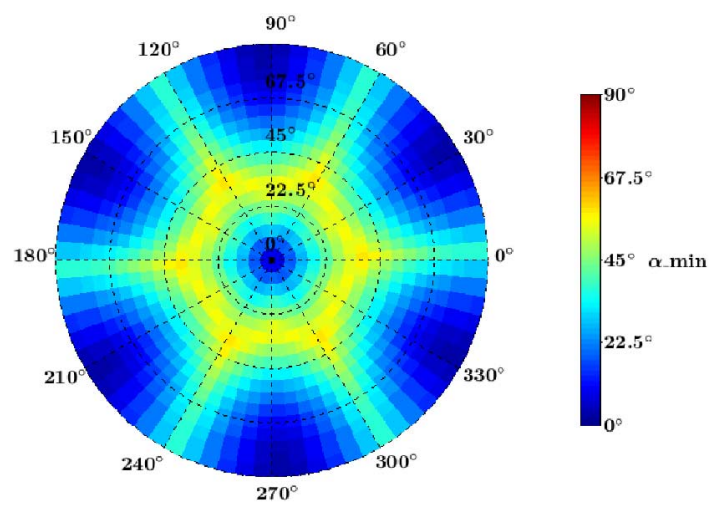

Fig. 4. 3-D theoretical ambiguity spectrum of the sensor $A B$ with $d_{E}^{A B}=$ $0.57 \lambda^{A}$ as a function of $\phi$ and $\theta$.

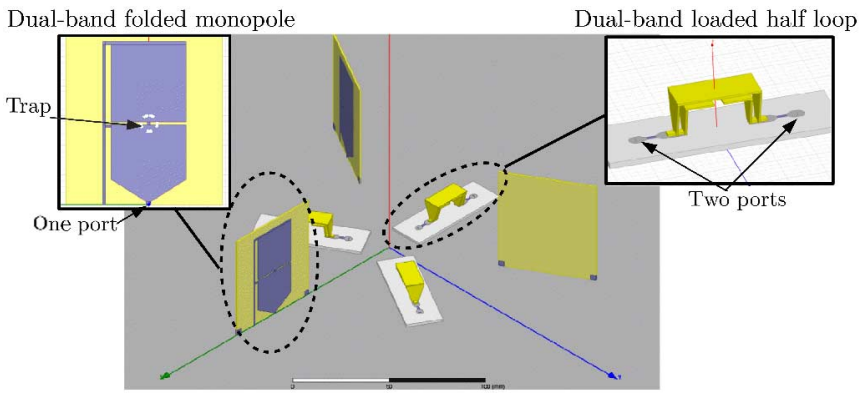

Fig. 5. Dual-band vector sensor using antennas spatially distributed and diversely polarized.

where $\odot$ is the element-wise multiplication operator, $\boldsymbol{r}_{E_{z i}^{A B}}$ and $r_{H_{x y i}^{A B}}$ denote the vectors associated with the position of the $i$ th dual-band electric and magnetic dipoles, respectively. The ambiguity analysis is carried out for $d_{E}^{A B}=2 d_{H}^{A B}$. Angular ambiguities appear when $d_{E}^{A B}=0.57 \lambda^{A}$ at $\theta=90^{\circ}$ and at $\phi \in\left[30^{\circ}, 90^{\circ}, 150^{\circ}, 210^{\circ}, 270^{\circ}, 330^{\circ}\right]$, where $\lambda^{A}$ is the smallest wavelength in the frequency band $A$. Fig. 4 gives the 3-D ambiguity spectrum for $d_{E}^{A B}=0.57 \lambda^{A}$. A singularity in the azimuth angle estimation exists and consequently, an angular ambiguity may occur when the angles $\theta$ approach zero (and for all separation distances $d_{E}^{A B}$ ). However, this ambiguity has no effect on the DoA estimation due to the low $\Delta_{a r m s}(\phi, \theta)$ resulting from this singularity at $\theta=0^{\circ}$ angle.

In order to assess the estimation performances of this sensor topology, a realistic sensor is now proposed.

\section{Design of the Dual-Band Vector Sensor}

A dual-band vector sensor based on the topology sketched in Figs. 3 and 5 is proposed. It incorporates a finite-sized metallic plate $(800 \times 800 \mathrm{~mm})$ and six dual-band antennas for estimating the DoAs of incoming TM EM-fields in the 3-D half-space and in the two following GSM frequency bands: $A=(1710$ $1880 \mathrm{MHz})$ and $B=(890-960 \mathrm{MHz})$. Dual-band foldedmonopoles and dual-band loaded half-loops are chosen here for measuring the required EM-field components. According to the ambiguity analysis carried out in Section IV-B, the separation distances between these antennas are set as follows: $d_{E}^{A B}=85 \mathrm{~mm} \approx 0.53 \lambda ; d_{H}^{A B}=42.5 \mathrm{~mm} \approx 0.265 \lambda$.

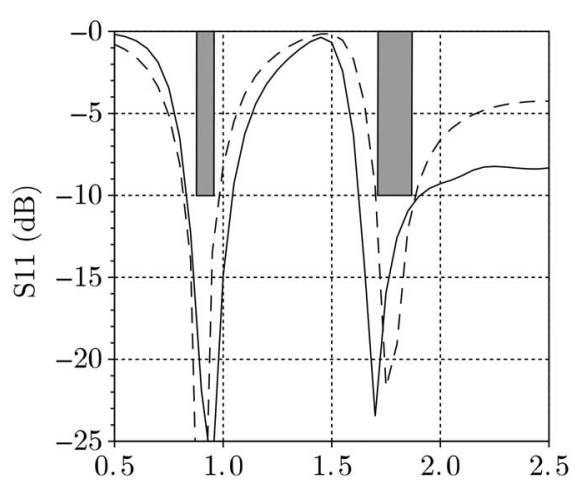

Fig. 6. Simulated reflection coefficient $S_{11}$ at the input of $E_{z 1}^{A B}$ (solid line) and $H_{x y 1}^{A B}$ (dashed line) with the technical requirement (gray regions).

Folded-monopoles are used here for achieving the required bandwidth of $10 \%$. They are preferred to quarter-wave monopoles [27] which offer a lower bandwidth for an equivalent antenna size. Note that these monopoles provide a natural protection against the electrostatic discharges. Moreover, they are loaded by resonant traps for dual-band operation. In order to measure the $\mathrm{H}$-field component, half-loops are chosen. These half-loops are loaded at each of their extremities by a capacitive effect (coupling with the bottom metallic plate) to reduce the antenna size. These compact antennas have convenient symmetric radiation pattern [10]. To achieve the dual-band operation, two half-loops are combined. The impedance matching is achieved by using a capacitive coupling. The EM analysis is performed by using a full-wave simulation software (HFSS). Consider the folded-monopole $E_{z 1}^{A B}$ located on the $0 x$-axis and directed along the $O z$-axis, and the half-loop $H_{x y 1}^{A B}$ located on the $O x$-axis and directed along the $O y$-axis. The EM simulation takes into account the eventual EM interactions between the constitutive antennas of the sensor. As depicted in Fig. 6, the frequency bands covered by the designed dual-band elements of the sensor and for a reflection coefficient lower than $-10 \mathrm{~dB}$ are:

- Band A: (1625-1904 MHz) (16\%) and band B: (830$1050 \mathrm{MHz})(23 \%)$ for the dual-band folded-monopoles;

- Band A: (1702-1890 MHz) (10\%) and band B: (815$980 \mathrm{MHz}$ ) (18\%) for the dual-band half-loops.

The required bandwidth is then achieved.

The normalized patterns of the $E_{z 1}^{A B}$ and $H_{x y 1}^{A B}$ antennas are reported in Fig. 7 ( $E$ - and $H$-plane refer to the $\mathrm{E}$ - and $\mathrm{H}$-field orientation, respectively). As expected the simulated radiation patterns are omnidirectional in the $H$ - and $E$-plane for the monopole and the half-loop (for $\theta \in\left[-90^{\circ} ; 90^{\circ}\right]$ ), respectively, and bidirectional in the $E$ - and $H$-plane for the monopole (for $\left.\theta \in\left[-90^{\circ} ; 90^{\circ}\right]\right)$ and the half-loop, respectively.

As shown in Fig. 7(c) and (d), the EM-fields scattered from the edge of the metallic support generate ripples in the E-plane radiation patterns of the antennas in the band $A$. In the $E$-plane at $\theta=90^{\circ}$, undesirable attenuations of about 5 and $10 \mathrm{~dB}$ in the two frequency bands are apparent. Moreover, an asymmetry occurs in radiation patterns due to EM scattering by the surrounding antennas in Fig. 7(a) and (c). The EM interaction between sensor radiating elements alters the radiation patterns 


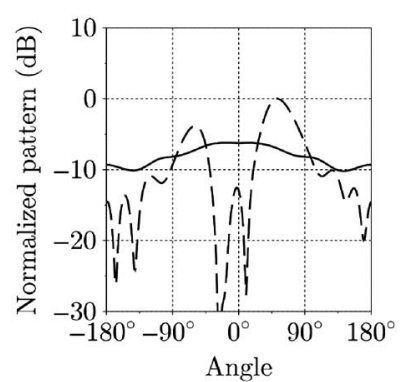

(a)

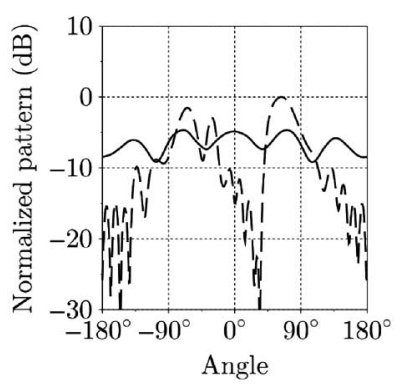

(c)

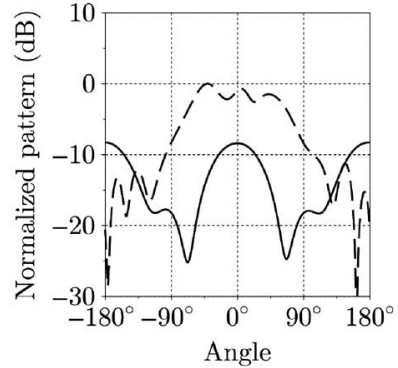

(b)

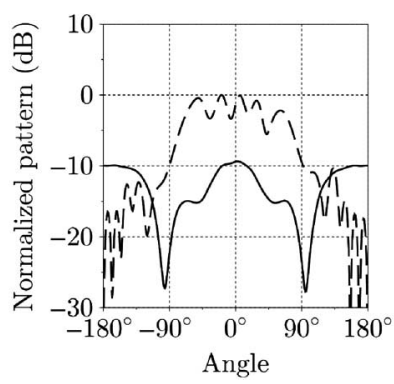

(d)
Fig. 7. Normalized patterns of $E_{z 1}^{A B}$ and $H_{x y 1}^{A B}$ in the $E$-plane (dashed line) and in the $H$-plane (solid line) (simulation results). (a) $E_{z 1}^{A B}$ at $925 \mathrm{MHz}$. (b) $H_{x y 1}^{A B}$ at $925 \mathrm{MHz}$. (c) $E_{z 1}^{A B}$ at $1795 \mathrm{MHz}$. (d) $H_{x y 1}^{A B}$ at $1795 \mathrm{MHz}$.

in the $H$-plane and provides ripples of about $4 \mathrm{~dB}$ on the directivity at 925 and $1795 \mathrm{MHz}$. However, the radiation patterns of the sensor antennas are acceptable since they allow, as it will be shown here, to obtain a good DoA estimation. The alteration in these patterns will be taken into account through the calibration process and its impact on the accuracy of the DoA estimation will be minimized.

\section{Accuracy of the DoA Estimation Computed From MUSIC Algorithm and Full-Wave EM Simulations}

The accuracy of the DoA estimation provided by the dualband sensor is computed from the MUSIC algorithm in the two frequency bands $A$ and $B$. The anechoic chamber used for measuring the sensor performances was designed for frequencies higher than $1000 \mathrm{MHz}$. Consequently the lowest frequency band $B$ was shifted up to $1000 \mathrm{MHz}$ for measurement purposes. The new frequency band is denoted by $B^{\prime}$. The estimation conditions are given as follows: $P_{r}=-105 \mathrm{dBW} \mathrm{m}^{-2}(\mathrm{PNR}=$ $\left.36 \mathrm{~dB} \mathrm{~m}^{-2}\right), \mathrm{N}=100$ snapshots per estimation, and $\mathrm{L}=20$ estimations per DoA. The rms error is computed at two frequencies, 1000 and $1800 \mathrm{MHz}$, and for $\phi \in\left[-60^{\circ} ; 60^{\circ}\right]$ by step of $1^{\circ}$ and for $\theta \in\left[0^{\circ} ; 90^{\circ}\right]$ by step of $5^{\circ}$. The vector sensor is calibrated as explained in Section II. Fig. 8 gives the simulated $\Delta_{\text {arms }}(\phi, \theta)$.

The DoA estimation is accurate in the 3-D half-space with a maximum rms error of $2.3^{\circ}$ at 1000 and $1800 \mathrm{MHz}$, located at $\theta$ close to $90^{\circ}$ and:

1) $\phi$ around $30^{\circ}$ and $330^{\circ}$ at $1000 \mathrm{MHz}$;

2) $\phi$ around $300^{\circ}, 0^{\circ}$, and $60^{\circ}$ at $1800 \mathrm{MHz}$.

No significant error is obtained in the estimation of the azimuth angles. The maximum rms error is lower than $1.1^{\circ}$ and

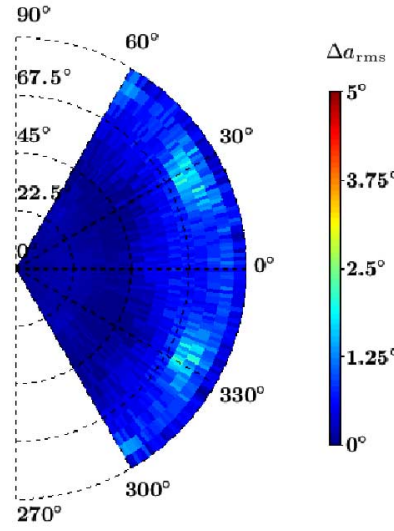

(a)

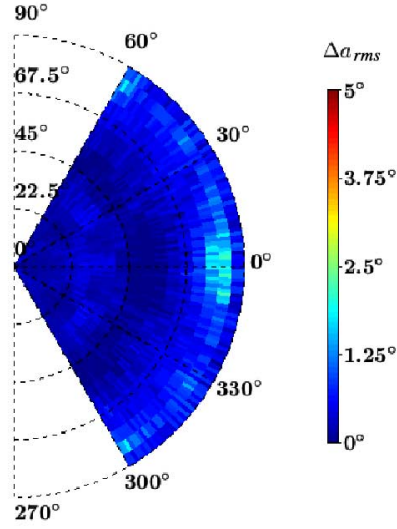

(b)
Fig. 8. Simulated $\Delta_{a \mathrm{rms}}(\phi, \theta)$ obtained with the dual-band vector sensor located on a $800 \times 800 \mathrm{~mm}$ metallic support at (a) 1000 and (b) $1800 \mathrm{MHz}$ with $P_{r}=-105 \mathrm{dBW} \mathrm{m}^{-2}\left(\mathrm{PNR}=36 \mathrm{~dB} \mathrm{~m}^{-2}\right), \mathrm{N}=100$, and $\mathrm{L}=20$.

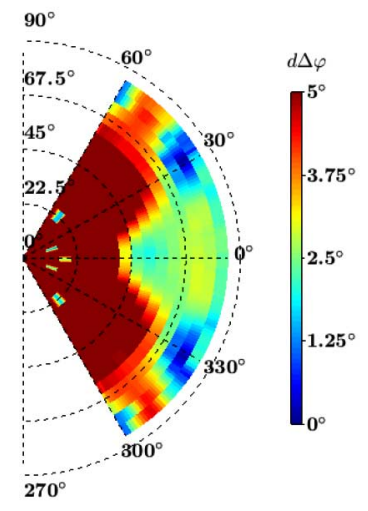

(a)

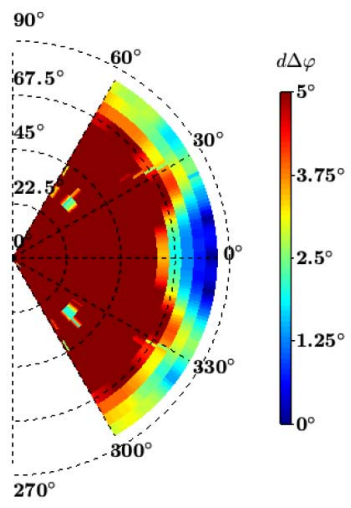

(b)
Fig. 9. Simulated $d \Delta \varphi(\phi, \theta)$ between the $E_{z i}^{A B}$ elements at (a) 1000 and (b) $1800 \mathrm{MHz}$.

$1.3^{\circ}$ at 1000 and $1800 \mathrm{MHz}$, respectively. In the topology of the dual-band sensor, the electric monopoles have been spatially distributed to improve the accuracy of the estimated $\theta$ angles. These monopoles provide an additional phase information at the outputs of the sensor, as defined in (6) and consequently, the estimation of the elevation angles is more accurate. The angles for which the DoA estimation errors is high can be determined by analyzing the variation of the phase differences between the $E_{z}^{A B}$ elements. Fig. 9 shows the mean variation of the phase differences $d \Delta \varphi(\phi, \theta)$ between $E_{z}^{A B}$ elements at 1000 and $1800 \mathrm{MHz}$.

These phase differences are computed from the mean phase shifts $\Delta \varphi(\phi, \theta)$ between electric antennas given by

$$
\Delta \varphi(\phi, \theta)=\frac{1}{M_{E}} \sum_{i=1}^{M_{E}}\left|\delta \varphi_{i}(\phi, \theta)\right|
$$

where $M_{E}$ denotes the number of electric antennas and $\delta \varphi_{i}\left(\phi, \theta_{n}\right)$ the $i$ th phase shift with

$$
\begin{aligned}
& \delta \varphi_{1}(\phi, \theta)=\varphi_{1}(\phi, \theta)-\varphi_{2}(\phi, \theta) \\
& \delta \varphi_{2}(\phi, \theta)=\varphi_{1}(\phi, \theta)-\varphi_{3}(\phi, \theta) \\
& \delta \varphi_{3}(\phi, \theta)=\varphi_{2}(\phi, \theta)-\varphi_{3}(\phi, \theta) .
\end{aligned}
$$




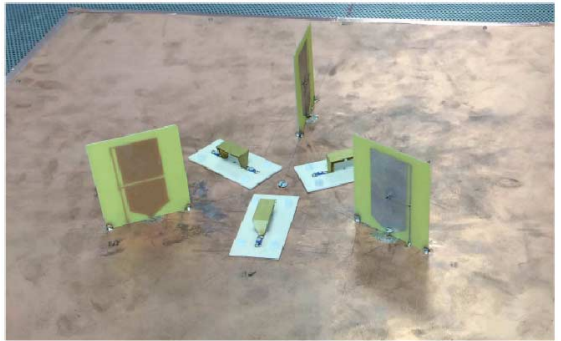

Fig. 10. Prototype of the dual-band vector sensor.

In (8), $\varphi_{1}, \varphi_{2}$, and $\varphi_{3}$ correspond to the phases at the output of $E_{z 1}^{A B}, E_{z 2}^{A B}$, and $E_{z 3}^{A B}$, respectively. The variation of $\Delta \varphi\left(\phi, \theta_{n}\right)$ is then derived according to the elevation angles

$$
d \Delta \varphi\left(\phi, \theta_{n}\right)=\left|\Delta \varphi\left(\phi, \theta_{n}\right)-\Delta \varphi\left(\phi, \theta_{n+1}\right)\right|
$$

where $n$ index indicates the $n$th elevation angle. The higher the phase variation, the better the estimation accuracy. As shown in Fig. 9, the areas with low phase variations are similar (as expected) to the ones derived from the computation of the rms errors (see Fig. 8). The dual-band vector sensor provides higher accuracy than the single-band reference vector sensor described in Section III. As a matter of fact the measurement of the incoming EM-field components by six distributed antennas improves significantly the estimation accuracy and reduces the estimation errors when $\theta$ is close to $90^{\circ}$. This improvement is due to the higher number of antennas and to the wider aperture provided by the topology sensor of six antennas. To analyze the sensor sensitivity, the accuracy of the DoA estimation is now computed for several received power densities: $P_{r}=-100,-105,-110$, and $-115 \mathrm{dBW} \mathrm{m}^{-2}$ which correspond to $\mathrm{PNR}=41,36,31$, and $26 \mathrm{~dB} \mathrm{~m}^{-2}$, respectively. This computation is performed for $\phi=30^{\circ}$ and $\phi=0^{\circ}$ (maximum error on the DoA estimation at 1000 and $1800 \mathrm{MHz}$, respectively) and for $\theta \in\left[0^{\circ} ; 90^{\circ}\right]$. The resulting rms errors are given in Fig. 14. For an estimation accuracy of $5^{\circ}$, the simulated sensitivity is found to be $P_{\text {sensi }}=-110 \mathrm{dBW} \mathrm{m}^{-2}$ $\left(\mathrm{PNR}=31 \mathrm{~dB} \mathrm{~m}^{-2}\right)$ at 1000 and $1800 \mathrm{MHz}$. The sensitivity of the reference sensor at $2400 \mathrm{MHz}$ is $P_{\text {sensi }}=-95 \mathrm{dBW} \mathrm{m}^{-2}$. By translating the performances of the dual-band sensor to $2400 \mathrm{MHz}$ (assuming a constant gain) the computed sensitivity are $P_{\text {sensi }}=-107.5 \mathrm{dBW} \mathrm{m}^{-2}$ in the band $A$ and $P_{\text {sensi }}=$ $-102.5 \mathrm{dBW} \mathrm{m}^{-2}$ in the band $B^{\prime}$. The proposed sensor is then more sensitive than the reference sensor with a sensitivity improvement of (at least) $7 \mathrm{~dB}$.

\section{EXPERIMENTAL VALIDATION}

A prototype of the dual-band sensor designed in Section IV has been manufactured (see Fig. 10) and measured. The measurement is performed at two operating frequencies, 1000 and $1800 \mathrm{MHz}$, in an anechoic chamber with the following estimation conditions: an incident power density $P_{r}=$ $-105 \mathrm{dBW} \mathrm{m}^{-2}$ which corresponds to $\mathrm{PNR}=36 \mathrm{~dB} \mathrm{~m}^{-2}$, $\mathrm{N}=100$ snapshots per DoA estimation, and $\mathrm{L}=20$ estimations per DoA. The EM-field is transmitted by a fixed horn antenna and illuminates the vector sensor placed on a rotating

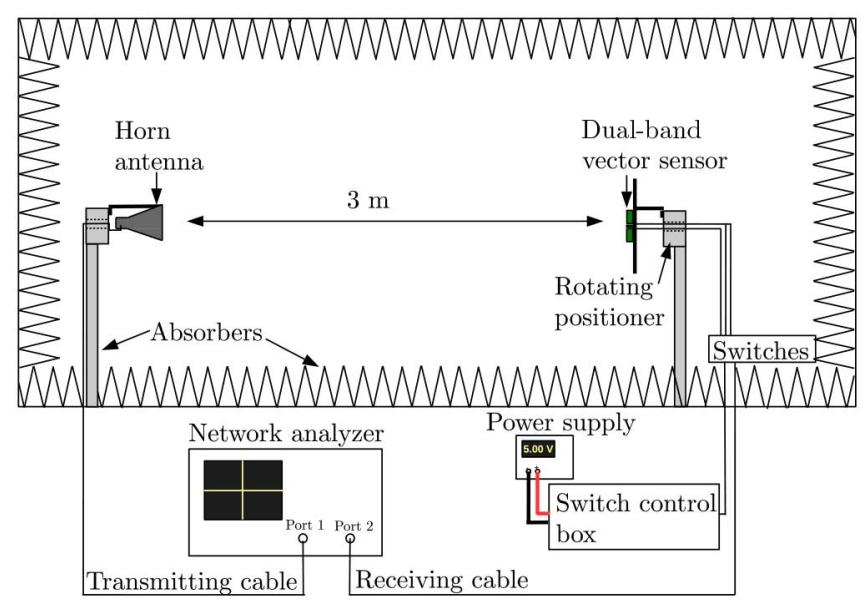

Fig. 11. Measurement setup.

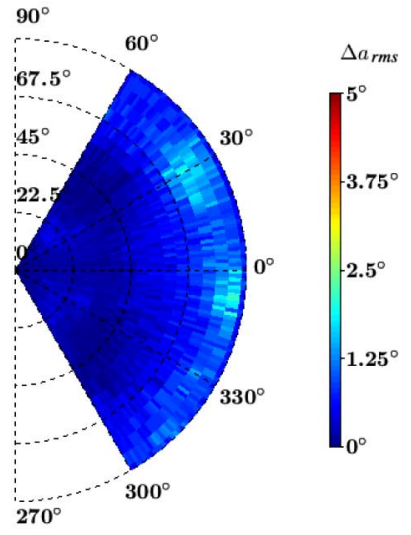

(a)

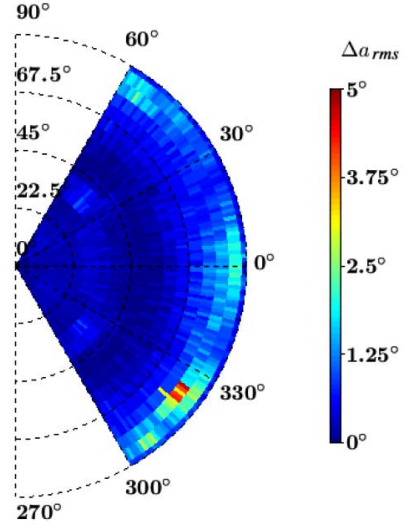

(b)
Fig. 12. Measured $\Delta_{a \mathrm{rms}}(\phi, \theta)$ obtained with the dual-band vector sensor located on a $800 \times 800 \mathrm{~mm}$ metallic support at (a) 1000 and (b) $1800 \mathrm{MHz}$ with $P_{r}=-105 \mathrm{dBW} \mathrm{m}^{-2}\left(\mathrm{PNR}=36 \mathrm{~dB} \mathrm{~m}^{-2}\right), \mathrm{N}=100$, and $\mathrm{L}=20$.

positioner. The vector analyzer Anritsu 37397D measures the amplitude and phase of the S-parameters at each port of the sensor antennas (see Fig. 11). As mentioned in Section II, eventual amplitude and phase distortions have been taken into account in the calibration process by measuring the radiation patterns of the sensor. Fig. 12 reports the measured rms errors of the DoA estimation in the two frequency bands. The estimation errors obtained from numerical simulations (see Fig. 8) and experimental results (see Fig. 12) are in good agreement. The estimation error differs at $1800 \mathrm{MHz}$ for $\theta=80^{\circ}$ and $\phi=315^{\circ}$. The maximum rms errors are $2.1^{\circ}$ and $4.7^{\circ}$ at 1000 and $1800 \mathrm{MHz}$, respectively. Some slight variations of the sensitive angular areas due to the low phase variation are apparent. They are located at $\theta$ close to $90^{\circ}$ and:

1) $\phi$ around $350^{\circ}$ and $330^{\circ}$ at $1000 \mathrm{MHz}$;

2) $\phi$ around $315^{\circ}, 0^{\circ}$, and $60^{\circ}$ at $1800 \mathrm{MHz}$.

For explaining these results, Fig. 13 shows $d \Delta \varphi(\phi, \theta)$ as a function of $\theta$.

$d \Delta \varphi(\phi, \theta)$ is altered with respect to the simulated phase variation given in Fig. 9. At $1000 \mathrm{MHz}, d \Delta \varphi(\phi, \theta)$ for $\theta$ close to $90^{\circ}$ and $\phi$ around $330^{\circ}$ increased whereas for $\phi$ close to $30^{\circ}$ a low variation $\left(d \Delta \varphi(\phi, \theta)<2^{\circ}\right)$ is maintained. This 


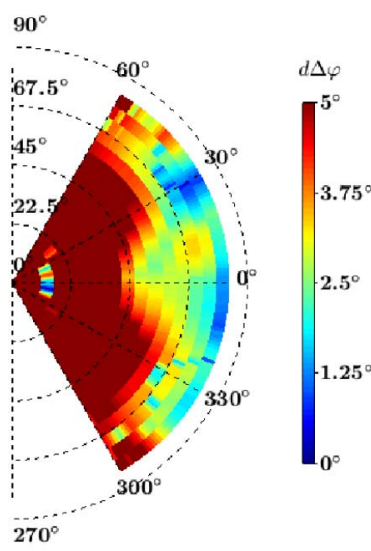

(a)

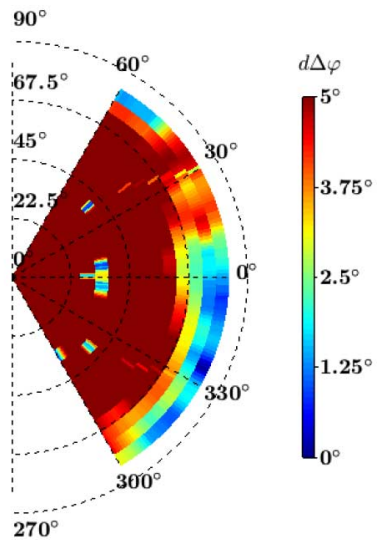

(b)
Fig. 13. Measured $d \Delta \varphi(\phi, \theta)$ at the output of the monopoles $E_{z i}^{A B}$ at (a) 1000 and (b) $1800 \mathrm{MHz}$.

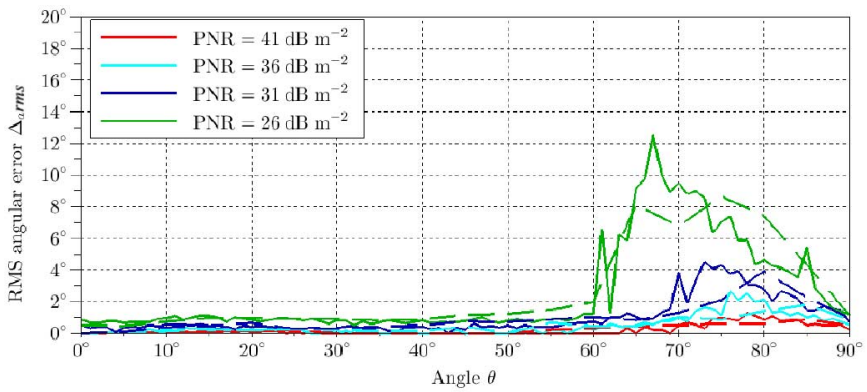

(a)

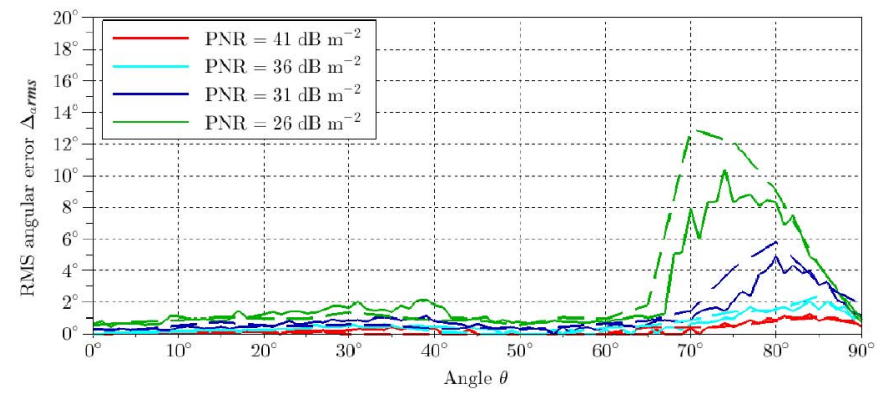

(b)

Fig. 14. Measured (dashed lines) and simulated (solid lines) $\Delta_{a \mathrm{rms}}(\phi, \theta)$ according to $\theta$ with (a) $\phi=30^{\circ}$ at $1000 \mathrm{MHz}$ and (b) $\phi=0^{\circ}$ at $1800 \mathrm{MHZ}$ for $\mathrm{PNR}=41,36,31$, and $26 \mathrm{~dB} m^{-2} ; \mathrm{N}=100$; and $\mathrm{L}=20$, in the worst case (azimuth angles of the maximum DoA error).

modification yields a new sensitive area for $\theta$ close to $90^{\circ}$ and for $\phi$ close to $350^{\circ}$ [see Fig. 13(a)]. At $1800 \mathrm{MHz}$, the maximum rms error occurs for $\phi$ close to $315^{\circ}$ because there is a reduction of $d \Delta \varphi(\phi, \theta)$ in this direction [see Fig. 13(b)].

Fig. 14 gives the measured and simulated estimation errors in the elevation plane with $\phi=30^{\circ}$ and $\phi=0^{\circ}$ at 1000 and $1800 \mathrm{MHz}$. The measured and simulated rms errors are in good agreement. The sensitivities are identical with $P_{\text {sensi }}=$ $-110 \mathrm{dBW} \mathrm{m}^{-2}$ for a maximum rms error of about $5^{\circ}$.

\section{Toward Multiband Vector Sensors}

To extend the number of operating frequency bands, multiband antennas could be used in the vector sensors.

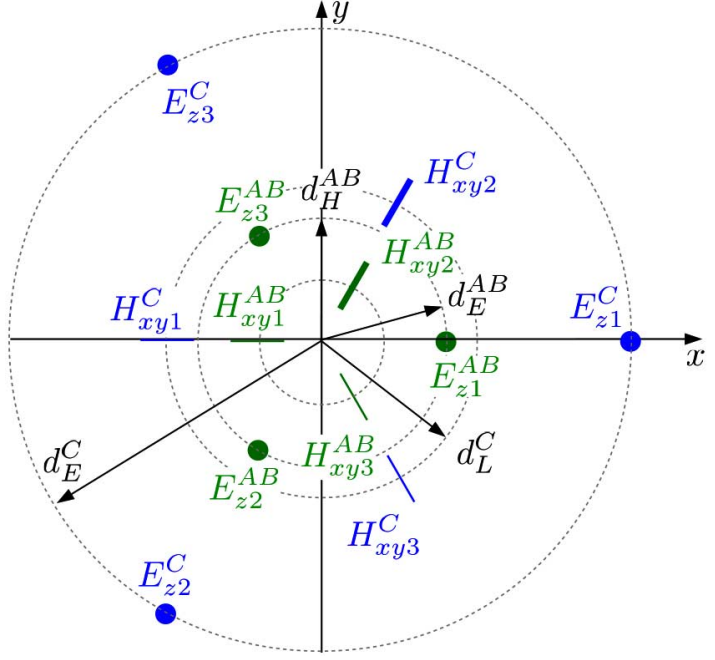

Fig. 15. Tri-band sensor using six distributed single-band antennas (blue) operating in the band $C$ and six distributed dual-band antennas (green) operating in the bands $A$ and $B$.

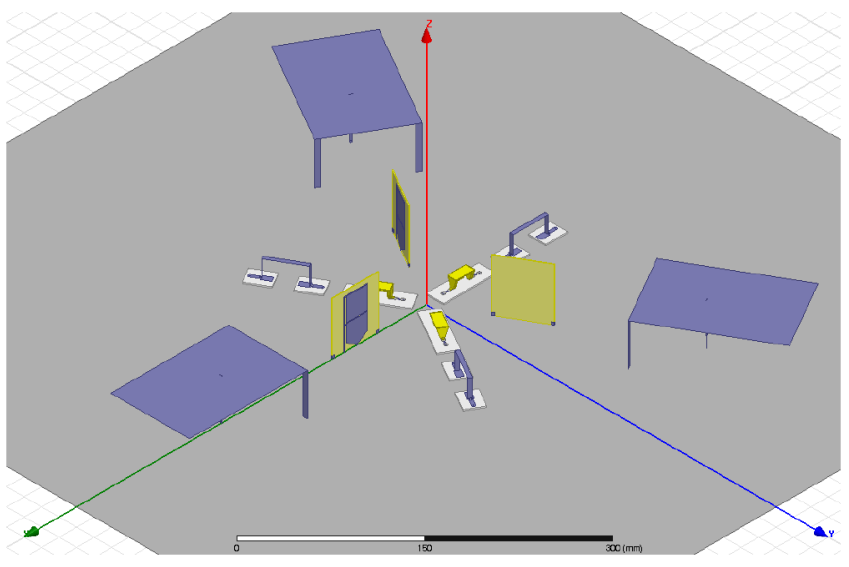

Fig. 16. Tri-band vector sensor using antennas spatially distributed and diversely polarized.

As above-mentioned the distance between the antennas has to be large enough to achieve an accurate DoA estimation but, this distance must be small enough for avoiding angular ambiguities.

Consider that a third operating frequency band $C=(400-$ $430 \mathrm{MHz}$ ) has to be added to the dual-band vector sensor. Following the design methodology reported in Section IV-A, a tri-band sensor architecture is proposed in Fig. 15.

As shown in Fig. 16, this new vector sensor uses three loaded half-loops $\left(H_{x y i}^{C}\right)$ for measuring the H-field along the $O x$ and $O y$-axes, and three planar inverted folded antennas (PIFA) $\left(E_{z i}^{C}\right)$ for measuring the E-field along the $z$-axis. The separation distances between antennas are denoted by $d_{H}^{C}$ and $d_{E}^{C}$. The ratio between these two distances is set to $d_{E}^{C} / d_{H}^{C}=2$ to avoid strong mutual EM interactions (see Section IV-B). The maximum distance $d_{\mathrm{AMB}}$ is the same as defined in Section IV. The distance $d_{E}^{C}$ and $d_{H}^{C}$ are set to $235 \mathrm{~mm} \approx 0.33 \lambda$ and $117.5 \mathrm{~mm}$ $\approx 0.165 \lambda$, respectively.

Fig. 17 gives the reflection coefficient $S_{11}$ at the input port of the antennas $E_{z 1}^{C}$ and $H_{x y 1}^{C}$. For a reflection coefficient lower 


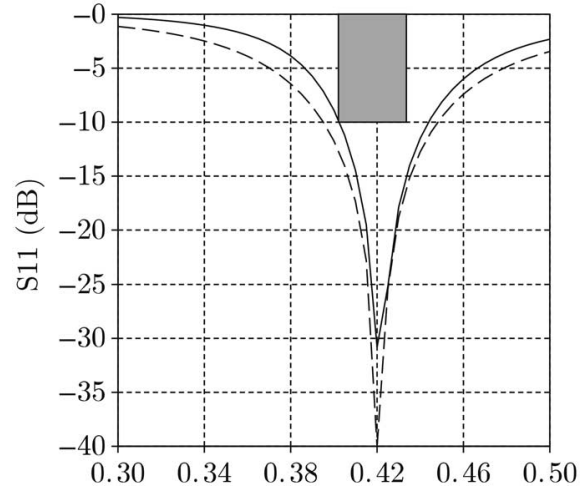

Fig. 17. Reflection coefficient $\mathrm{S}_{11}$ of the PIFA antenna $E_{z 1}^{C}$ (solid line) and the loaded half-loop $H_{x y 1}^{C}$ (dashed line) with the technical requirement (gray region).

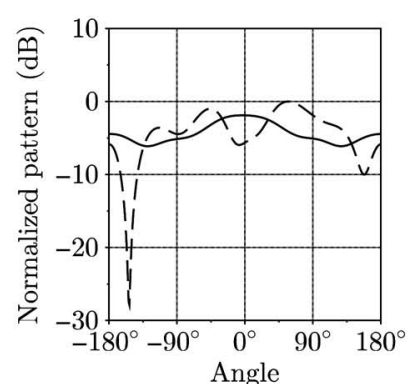

(a)

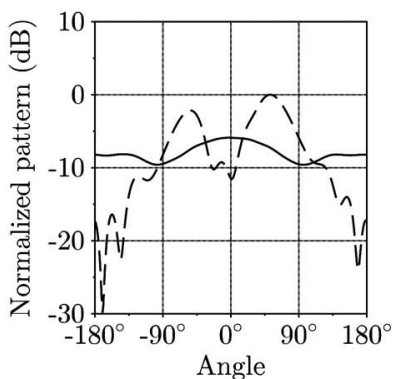

(c)

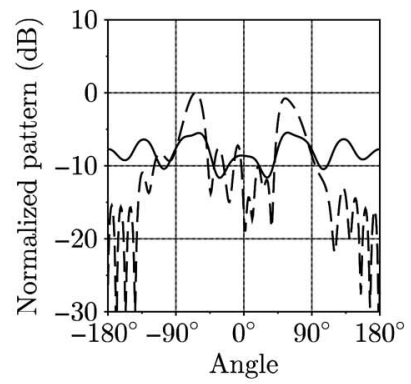

(e)

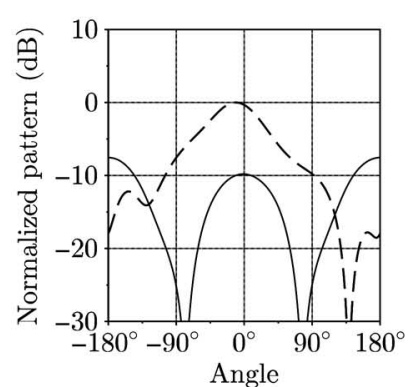

(b)

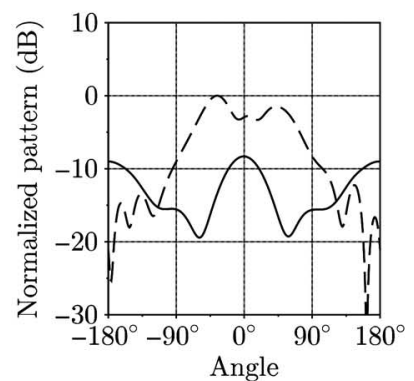

(d)

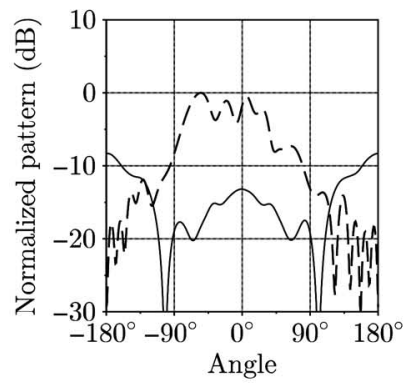

(f)
Fig. 18. Normalized patterns of (a) $E_{z 1}^{C}$ at $415 \mathrm{MHz}$; (b) $H_{x y 1}^{C}$ at $415 \mathrm{MHz}$; (c) $E_{z 1}^{A B}$ at $925 \mathrm{MHz}$; (d) $H_{x y 1}^{A B}$ at $925 \mathrm{MHz}$; (e) $E_{z 1}^{A B}$ at $1795 \mathrm{MHz}$; and (f) $H_{x y 1}^{A B}$ at $1795 \mathrm{MHz}$ simulated in the $E$-plane (dashed line) and $H$-plane (solid line).

than $-10 \mathrm{~dB}$, the operating frequency bands are (in presence of all the antennas $E_{z}^{A B}$ and $\left.H_{x y}^{A B}\right)$ :

1) (401-444 MHz) for the PIFA antennas;

2) (395-448 MHz) for the loaded half-loops.

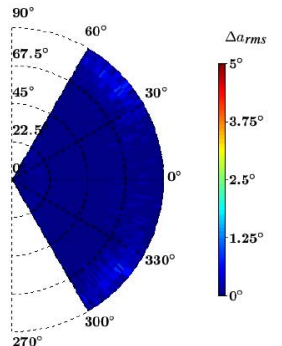

(a) (b)

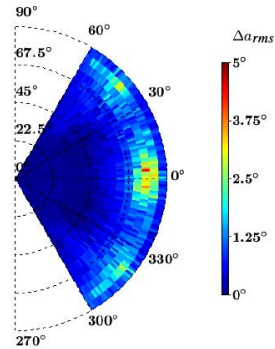

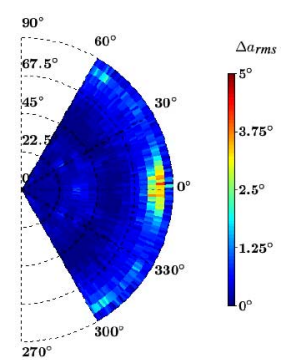

(c)
Fig. 19. Simulated $\Delta_{a \mathrm{rms}}$ obtained with the tri-band vector sensor located on a $800 \times 800 \mathrm{~mm}$ metallic support: $\mathrm{PNR}=36 \mathrm{~dB} \mathrm{~m}^{-2}, \mathrm{~N}=100$, and $\mathrm{L}=20$. (a) 415; (b) 925; and (c) $1795 \mathrm{MHz}$

The normalized patterns of the antennas $E_{z 1}^{A B}, E_{z 1}^{C}$ and $H_{x y 1}^{A B}, H_{x y 1}^{C}$ in the presence of all sensor antennas are shown in Fig. 18.

Ripples observed on the radiation pattern at 925 and $1795 \mathrm{MHz}$ due to the scattering of the EM-field from the edge of the metallic support are present. These ripples are weak at $415 \mathrm{MHz}$ due to the small electric size of this support in the band $C$. Note weak distortions of the radiation patterns occur in the bands $A$ and $B$ due to the presence of the low frequencies antennas.

Fig. 19 gives the simulated $\Delta_{a r m s}(\phi, \theta)$ for the tri-band sensor. The accuracy of the DoA estimation is computed at the three following frequencies: 415,925 , and $1795 \mathrm{MHz}$. The estimation conditions are identical to those given in Section IV-B.

At $415 \mathrm{MHz}$, the simulated estimation accuracy is lower than $1^{\circ}$ over the 3-D half-space. At 925 and $1795 \mathrm{MHz}$, the maximum rms error is $4.7^{\circ}$ and $4.1^{\circ}$, respectively. As expected, the presence of the low frequencies antennas alters slightly the DoA estimation performances in the frequency bands $A$ and $B$ by generating undesirable masking in the sensitive angular areas previously identified. Nevertheless, the tri-band vector sensor provides an acceptable estimation accuracy (lower than $5^{\circ}$ ) of the DoA in the 3-D half-space, and it shows that the proposed Russian dolls' topology may be convenient for designing multiband vector sensors using antennas spatially distributed and diversely polarized.

The proposed approach allows adjusting separation distances between sensor antennas for guaranteeing an accurate DoA estimation and avoiding angular ambiguities. Nevertheless, the combination of single-band vector sensors for designing multiband sensors may yield undesirable masking effects at high frequencies due to the sensor constitutive antennas. The minimization of this effect is a crucial challenge for the design of multiband vector sensors.

\section{CONCLUSION}

For the first time a dual-band vector sensor using antennas spatially distributed and diversely polarized is proposed for an accurate DoA estimation of incoming EM-fields in the 3-D half-space. This compact sensor $(\lambda / 4$ in radius and $\lambda / 5.5$ in height at the lowest operating frequency) is composed of six small dual-band distributed antennas to cover two frequency 
bands: three dual-band folded-monopoles and three dual-band loaded half-loops. The antennas are located on a finite-sized metallic plate. An original sensor topology is proposed and criteria are defined to describe its performances. A prototype has been manufactured and measured for experimental validation. The investigation based on the study of the phase variation between the sensor antennas is proposed to identify the angular areas where the estimation is more sensitive to the noise. Finally, a low-frequency single-band sensor has been incorporated into the dual-band sensor to illustrate the feasibility of multiband vector sensors. The simulated performances of the tri-band sensors have been predicted from full-wave EM simulations and show that the integration of the third operating frequency band generates a slight degradation of the dual-band sensor performances.

\section{REFERENCES}

[1] T. E. Tuncer and B. Friedlander, Classical and Modern Directionof-Arrival Estimation, Elsevier, Ed. New York, NY, USA: Academic, 2009.

[2] P. J. D. Gething, Radio Direction Finding and Superresolution, P. Peregrinus Ltd. on behalf of the Institution of Electrical Engineers, 1991.

[3] G. F. Hatke, "Conditions for unambiguous source location using polarization diverse arrays," in Proc. Asilomar Conf. Signals Syst. Comput., 1993, pp. $1365-1369$.

[4] L. Lo Monte, B. Elnour, and D. Erricolo, "Distributed 6d vector antennas design for direction of arrival applications," in Proc. Int. Conf. Electromagn. Adv. Appl. (ICEAA), 2007, pp. 431-434.

[5] L. Lo Monte, B. Elnour, D. Erricolo, and A. Nehorai, "Design and realization of a distributed vector sensor for polarization diversity applications," in Proc. Int. Waveform Diversity Des. Conf., 2007, pp. 358-361.

[6] L. Sun, C. Li, Y. Lu, and G. Ou, "Distributed vector sensor cross product added with MUSIC for direction of arrival estimation," in Proc. Asia-Pac. Symp. Electromagn. Compat. (APEMC), 2010, pp. 1354-1357.

[7] A. Chabory, C. Morlaas, and B. Souny, "Efficiency characterization of vector-sensor antennas with distributed elements for $3 \mathrm{D}$ direction finding," in Proc. IEEE-APS Top. Conf. Antennas Propag. Wireless Commun., 2011, pp. 819-822.

[8] C. M. S. See and A. Nehorai, "Source localization with distributed electromagnetic component sensor array processing," in Proc. 7th Int. Symp. Signal Process. Its Appl., 2003, vol. 1, pp. 177-180.

[9] K. T. Wong and X. Yuan, "Vector cross-product direction-finding" with an electromagnetic vector-sensor of six orthogonally oriented but spatially noncollocating dipoles/loops," IEEE Trans. Signal Process., vol. 59, no. 1, pp. 160-171, Jan. 2011.

[10] J. Lominé, C. Morlaas, and H. Aubert, "Novel vector sensors design with three co-located or distributed elements for the 3D DoA estimation," Prog. Electromagn. Res. B, vol. 57, pp. 207-220, 2014.

[11] A. Nehorai and E. Paldi, "Vector-sensor array processing for electromagnetic source localization," IEEE Trans. Signal Process., vol. 42, no. 2 , pp. 376-398, Feb. 1994.

[12] X. Yuan, K. T. Wong, Z. Xu, and K. Agrawal, "Various compositions to form a triad of collocated dipoles/loops, for direction finding and polarization estimation," IEEE Sensors J., vol. 12, no. 6, pp. 1763-1771, Apr. 2012.

[13] M. Hirari and M. Hayakawa, "DoA estimation using blind separation of sources," in Proc. IEEE Signal Process. Workshop Higher-Order Statist., 1997, pp. 311-315.

[14] H. S. Mir, J. D. Sahr, and C. M. Keller, "Source localization using airborne vector sensors," in Proc. IEEE Int. Conf. Acoust. Speech Signal Process. (ICASSP), 2005, vol. 4, pp. iv/1033-iv/1036.

[15] H. S. Mir and J. D. Sahr, "Passive direction finding using airborne vector sensors in the presence of manifold perturbations," IEEE Trans. Signal Process., vol. 55, no. 1, pp. 156-164, Jan. 2007.

[16] K. T. Wong and A. K. Y. Lai, "Inexpensive upgrade of base-station dumb antennas by two magnetic loops for "blind" adaptive downlink beamforming," IEEE Antennas Propag. Mag., vol. 47, no. 1, pp. 189-193, Jul. 2005.

[17] S. Appadwedula and C. M. Keller, "Direction-finding results for a vector sensor antenna on a small UAV," in Proc. 4th IEEE Workshop Sensor Array Multichannel Process., 2006, pp. 74-78.
[18] J. Lominé, C. Morlaas, and H. Aubert, "Method for vector sensor design based on a spherical mode approach for 3D DoA estimation," in Proc. 7th Eur. Conf. Antennas Propag. (EuCAP), 2013, pp. 237-241.

[19] R. O. Schmidt, "Multiple emitter location and signal parameter estimation," IEEE Trans. Antennas Propag., vol. 34, no. 3, pp. 276-280, Mar. 1986.

[20] M. Hurtado and A. Nehorai, "Performance analysis of passive lowgrazing-angle source localization using diversely polarized arrays," IEEE Trans. Aerosp. Electron. Syst., vol. 43, no. 2, pp. 780-789, Aug. 2007.

[21] S. Chandran, Advances in Direction-of-Arrival Estimation. Norwood, MA, USA: Artech House, 2005.

[22] A. N. Lemma, E. F. Deprettere, and A. J. van der Veen, "Experimental analysis of antenna coupling for high-resolution DoA estimation algorithms," in Proc. 2nd IEEE Workshop Signal Process. Adv. Wireless Commun. (SPAWC), 1999, pp. 362-365.

[23] W. Guibao, T. Haihong, S. Jia, G. Xiaolu, Z. Cao, and W. Lanmei, "Mutual coupling calibration for electromagnetic vector sensor array," in Proc. 10th Int. Symp. Antennas Propag. EM Theory (ISAPE), 2012, pp. 261-264.

[24] A. Khallaayoun, R. J. Weber, and Y. Huang, "A blind iterative calibration method for high resolution DoA estimation," in Proc. Mil. Commun. Conf. (MILCOM), 2011, pp. 199-204.

[25] D. Spielman, A. Paulraj, and T. Kailath, "Performance analysis of the MUSIC algorithm," in Proc. IEEE Int. Conf. Acoust. Speech Signal Process. (ICASSP), 1986, vol. 11, pp. 1909-1912.

[26] G. Maral, Satellite Communications Systems: Systems, Techniques and Technology, 5th ed. Hoboken, NJ, USA: Wiley, 2009.

[27] W. L. Stutzman and G. A. Thiele, Antenna Theory and Design, 3rd ed. Hoboken, NJ, USA: Wiley, 2012.

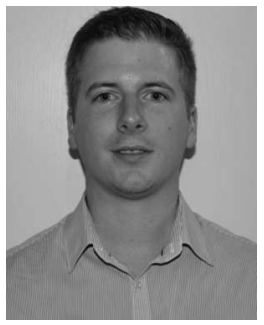

Jimmy Lominé was born in Dinan, France, on October 1985. He received the Engineering degree in electrical engineering and signal processing from the University of Rennes 1, Rennes, France, in 2008, and the Ph.D. degree in electrical engineering from the Institut National Polytechnique (INPT), Toulouse, France, in 2014.

He was an Electrical Engineer with companies of Engineering and Technology Consultancy, from September 2009 to October 2011, working with Thales and Rockwell Collins France, Blagnac, France. Since November 2014, he has been working as an Antenna and System Engineer with the Department of Navigation and Surveillance System Engineering, Rockwell Collins France. His research interests include the antenna design, the electromagnetic modeling, and the navigation and radar systems.

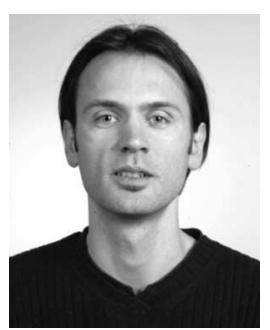

Christophe Morlaas received the Ph.D. degree in electronics from ENSAE (Superior National School of Aeronautical and Spatial), Malakoff, France, in 2000.

He was a System Engineer on spatial programs until 2002. Since 2002, he has been a Teacher/Researcher with the TELECOM Laboratory, ENAC (National School of Civil Aviation), Toulouse, France, in electromagnetism and antenna domains. His research interests include aeronautical communication and navigation systems, electromagnetic modeling, propagation models, and antenna design.

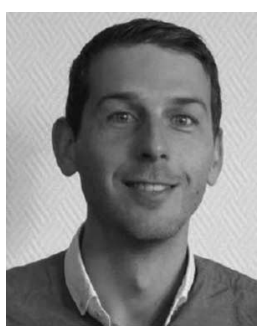

Christophe Imbert was born in Manosque, France, on April 1978. He received the Engineering degree in electrical engineering from ENAC (National School of Civil Aviation), Toulouse, France, in 2002.

Since February 2005, he has been working as a System Engineer with the Department of Navigation and Surveillance System Engineering, Rockwell Collins France, Blagnac, France, and is involved in the development of airborne direction finders and radar systems. 


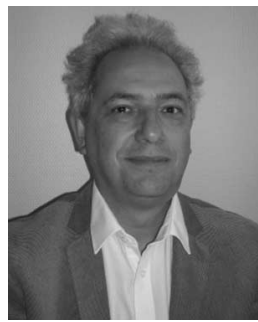

Hervé Aubert (M'94-SM'99) was born in Toulouse, France, on July 1966. He received the Eng.Dipl. and $\mathrm{Ph} . \mathrm{D}$. (Hons.) degrees in electrical engineering from the Institut National Polytechnique (INPT), Toulouse, France, in 1989 and 1993, respectively.

Since February 2001, he has been a Professor with INPT. He has joined the Laboratory for the Analysis and Architecture of Systems (LAAS), National Center for Scientific Research (CNRS), Toulouse, France, in February 2006. Since January 2015, he has been the Head of the Micro- and Nano-systems for Wireless Communications Research Group, LAAS-CNRS. From April 1997 to March 1998, he was a Visiting Associate Professor at the School of Engineering and Applied Science, University of Pennsylvania, Philadelphia, PA, USA. He was the Co-Chairman of the Electronics Laboratory, INPT from July 2001 to January 2005 and the Head of the Electromagnetics Research Group of this Laboratory from July 2002 to September 2005. Currently his research activities involve the electromagnetic modelling of complex (multi-scale) structures and Wireless Sensors Networks. He has authored or coauthored one book, two book chapters, 75 papers in refereed journals, and over 200 communications in International Symposium Proceedings. His research interests include integralequation and variational methods applied to electromagnetic wave propagation and scattering.

Dr. Aubert is a member of the Editorial Board of the International Journal of Microwave Science and Technology and the International Journal of Antennas and Propagation. He is the General Chairman of the European Microwave Week in 2015 (Paris, France). He has been expert for the French National Research Agency (ANR) since 2009 and for the European Commission since 2012. 